\title{
Geri Dönüştürülebilir Atıkların Materyallerine Göre Sınıflandırılması için Raspberry Pi Tabanlı Donanım Geliştirilmesi
}

\author{
Ali Sağlam ${ }^{1 *}$, Melike Taş², Nurdan Akhan Baykan ${ }^{3}$ \\ ${ }^{1}$ Konya Teknik Üniversitesi, Mühendislik ve Doğa Bilimleri Fakültesi, Bilgisayar Mühendisliği Bölümü, Konya, Türkiye (ORCID: 0000-0003-2980-9666) \\ ${ }^{2}$ Konya Teknik Üniversitesi, Mühendislik ve Doğa Bilimleri Fakültesi, Bilgisayar Mühendisliği Bölümü, Konya, Türkiye (ORCID: 0000-0003-1581-7008) \\ ${ }^{3}$ Konya Teknik Üniversitesi, Mühendislik ve Doğa Bilimleri Fakültesi, Bilgisayar Mühendisliği Bölümü, Konya, Türkiye (ORCID: 0000-0002-4289-8889)
}

( $1^{\text {st }}$ International Conference on Computer, Electrical and Electronic Sciences ICCEES 2020 - 8-10 Ekim 2020)

(DOI: 10.31590/ejosat. 802692)

ATIF/REFERENCE: Saglam, A. \& Taş, M. \& Baykan, N.A. (2020). Geri Dönüştürülebilir Atıkların Materyallerine Göre Sınıflandırılması için Raspberry Pi Tabanlı Donanım Geliştirilmesi. Avrupa Bilim ve Teknoloji Dergisi, (Özel Sayı), $30-38$.

$\ddot{\mathbf{O} z}$

Hem doğanın korunması hem de sürekli artan insan ihtiyaçları için gerekli olan ve doğada kısıtlı miktarda bulunan materyallerin takviye edilmesi için ortaya çıkan "geri dönüşüm” kavramı son yıllarda en önemli konulardan birisi olmuştur. Belirli bir geri dönüşüm işlemi sonucunda, "ham maddesi yeniden kullanılabilir hale getirilebilen atıklar" olarak bilinen geri dönüştürülebilir atıkların toplanması konusu dünya genelinde üst ve yerel yönetimlerin de ilgilendiği bir problem olmuştur. Bunun için belirli merkezlere geri dönüştürülebilir atıklar için özel kutular yerleştirilmekte ve insanlar geri dönüşüm konusunda teşvik edilmeye çalışılmaktadır. Bu çalışmada, geri dönüşüm projelerinde kullanılmak üzere kâğıt, cam ve plastik atıklarının geri dönüşüm kutuları içerisinde gerçek zamanlı olarak tespit edilebilmesi için gerekli elektronik malzemeler ve yazılımlar kullanılarak bir materyal tanıma sistemi geliştirilmektedir. Sistem geri dönüşüm kutusuna atılan geri dönüştürülebilir katı atıkların materyallerini tanıyan ve materyale göre kullanıcı hesabına ücret yükleyen bir simülasyon işlevi görmektedir. Geliştirilen donanım kamera, LCD ekran, LED, IR LED, devre tahtası ve jumper kablo gibi Raspberry Pi üzerine bağlanabilen elektronik cihazları da içermektedir. Materyallerin tanınması için gerekli yazılımının geliştirilmesi aşamasında; kâğıt, cam ve plastik materyallerini içeren 845 adet resim çalışma kapsamında hazırlanmış ve bunların 662 tanesi Tensorflow nesne tanıma kütüphanesi üzerinde eğitim için kullanılmıştır. Materyallerin geliştirilen donanım tarafından gerçek zamanlı olarak algılanması ve elde edilen nesne tanıma modelinin donanım üzerinde kullanılabilmesi için Raspberry Pi içerisine OpenCV bilgisayarlı görme kütüphanesi yüklenmiştir. En son olarak, geliştirilen donanım ilgili materyallere özel ayrılmış kutular üzerine sabitlenerek sistem gerçek zamanlı olarak çalışır hale getirilmiştir. Sistemin düzgün çalıştığını doğrulamak için kutu içerisine bazı atıklar atılmış ve LCD ekran üzerinde sonuçlar görüntülenmiştir.

\section{Development of Raspberry Pi Based Hardware for Classification of Recyclable Wastes According to Their Materials}

\begin{abstract}
The concept of "recycling", which emerged to reinforce the limited amount of materials in nature, which is necessary for both the protection of nature and the ever-increasing human needs, has been one of the most important issues in recent years. The issue of collecting recyclable wastes known as "the wastes whose raw materials can be reused" as a result of a certain recycling process has been a problem that the top and local governments interest around the world. For this, special boxes for recyclable wastes are placed in certain centers and people are encouraged to recycle. In this study, a material recognition system is developed by using the necessary electronic materials and software to detect paper, glass and plastic wastes in recycling bins in real-time to be used in recycling projects. The system functions as a simulation that recognizes the materials of recyclable solid wastes thrown into the recycling bin and charges
\end{abstract}

* Sorumlu Yazar: Konya Teknik Üniversitesi, Mühendislik ve Doğa Bilimleri Fakültesi, Bilgisayar Mühendisliği Bölümü, Konya, Türkiye, ORCID: 0000-0003-2980-9666, asaglam@ktun.edu.tr 
the user account according to the material. The developed hardware includes electronic devices that can be connected to the Raspberry Pi such as camera, LCD screen, LED, IR LED, breadboard and jumper cable. During the development of the software required for the recognition of the materials, 845 pictures including paper, glass and plastic materials were prepared within the scope of the study and 662 of them were used for training on the Tensorflow object recognition library. The OpenCV computer vision library has been loaded into the Raspberry Pi so that the materials can be detected in real-time by the developed hardware and the obtained object recognition model can be used on the hardware. Finally, the system has become works in real-time by fixing the developed hardware on boxes dedicated to the relevant materials. To verify that the system is working properly, some waste has been thrown into the boxes and the results are displayed on the LCD screen.

Keywords: Raspberry Pi, Recycling, Image Processing, Deep Learning, Object Recognition.

\section{Giriş}

Dünya genelinde süregelen nüfus artışı, kentleşme ve sanayileşmenin etkisiyle birlikte yoğun miktarda kaynak kullanılmakta ve bunun sonucunda da yüksek oranda atık oluşmaktadır. Her geçen gün artan atıklar, toplumu ve doğayı oldukça olumsuz derecede etkilemektedir [1]. Bütün atıkların çöp arazilerine bir daha kullanılmamak üzere terk edilmesi, özellikle plastik atıklar gibi doğada sonsuza kadar varlığını sürdürebilen atıkların bulunması, doğadaki canlılar için oldukça tehlike arz etmektedir [2], [3]. Bununla birlikte, bu çalışma kapsamında üzerinde durulan plastik, cam ve kâğıt gibi maddelerin temini sürecinde küresel 1sınma artmakta, enerji kaynakları tükenmekte, ormanlar büyük bir hızla yok olmakta ve atmosfer kirlenmektedir. Ayrıca bu kaynakların tekrar temini ve üretiminden dolayı ülkelerin ekonomisinde kayıplar oluşmaktadır [4].

Birçok kategoriden oluşan atık çeşitleri arasında katı atıklar kategorisine giren cam, plastik, kâğıt, metal ve karton gibi malzemelerin fiziksel, kimyasal ve biyolojik işlem ve süreçlerden geçirildikten sonra hammaddelerinin saf olarak tekrar ortaya çıkarılıp, yeniden kullanılabilir hale getirilmesi olayına "geri dönüşüm" denilmektedir [5], [6]. Bu şekilde yeniden kullanılabilir hale getirilebilen atıklara da "geri dönüştürülebilir atık" denilmektedir. Doğada uzun süre bozulmadan kalabilen cam, plastik ve kâğıt gibi maddelerden yapılan malzemeler en fazla tüketilen malzemeler olmakla birlikte, bu maddelerin geri dönüşümü de kolay olmaktadır. Bu atıkların geri dönüşüm işlemleri sürecinde harcanan enerji miktarı ham maddeden yeni malzeme oluşturulması esnasında harcanan enerji miktarına göre oldukça düşük seviyededir. Bu maddelerin geri dönüşümü ile yeni ham madde ihtiyacı azaltılmakta, doğal kaynaklar korunmakta, fazladan enerji tüketimi önlenmekte ve doğadaki canlılara zarar veren katı atık salınımı azaltılmaktadır [7].

Bugün doğayı ve ekonomisini korumak isteyen birçok ülke geri dönüşüm konusu üzerinde önemle durmakta, devlet kurumları ve yerel yönetimler işbirliği içerisinde bununla ilgili projeler geliştirmektedir [1], [8], [9]. Gelişmiş toplumlarda insanlar da geri dönüştürülebilir atıkların toplanması konusunda bilinçlendirilmekte ve teşvik edilmektedir. Geri dönüştürülebilir atıklar için yerleşim yerlerinin belirli noktalarına geri dönüşüm konteynerleri yerleştirilmekte ve buralardan geri dönüştürülebilir atıklar toplanmaktadır.

$\mathrm{Bu}$ çalışmada, insanların geri dönüştürülebilir atıkları ilgili konteynerlere atmalarını teşvik etmede kullanılabilecek, yani geri dönüştürülebilir atık ilgili konteynere atıldığında karşılığında kişiye özel karta para veya puan olarak yükleme yapabilmesinde kullanılabilecek projeler için, bir atık tanıma donanımı geliştirilmektedir. Bu kapsamda, kamera, LCD ekran ve hoparlör gibi veri girdisi ve çıktısı sağlayabilecek malzemelerden oluşan ve Raspberry Pi bilgisayarı üzerinde çalışan sistem, konteynerler üzerinde gerçek zamanlı olarak çalışabilir hale getirilmiştir. Ayrıca, donanımın gerekli işlevi yerine getirmesini sağlayan gömülü yazılım geliştirilmiş ve nesnelerin tanınması için eğitilmiştir. Cam, plastik ve kâğıt maddelerinden yapılmış olan malzemelerin bulunduğu resimler elde edilerek eğitim ve test aşamasında kullanılmıştır. Geliştirilen sistemde, kutular içerisine yerleştirilen Raspberry Pi 3 mini bilgisayarı, kamera, LED, IR alıcı-verici kızılötesi LED ve devre tahtası ile dış tarafa yerleştirilen LCD ekran jumper kablolar ile birbirine bağlanarak, kutuya atılan çöpün maddesine göre kullanıcıya bilgi sağlayan bir donanım geliştirilmiştir. LCD ekranda ilgili materyale göre kullanıcı hesabına bakiye transferi yapıldı̆̆ı gösterilmektedir. Bu donanım gerçek kullanıcı hesabı kullanmamakta; gerçek hayattaki kullanım için bir simülasyon özelliği göstermektedir.

\section{Materyal ve Metot}

\subsection{Raspberry Pi 3}

Raspberry Pi tek karttan oluşan bir mini bilgisayardır. Bir bilgisayar için gerekli olan işlemci, RAM bellek, giriş/çıkışlar gibi tüm birimler tek bir devre kartı üzerinde toplanmıştır. Küçük olması ve uyumlu yapısı sayesinde bu mini bilgisayarlar robotlar, akıllı ev sistemleri, otomatlar, iş makineleri ve üretim tezgâhları gibi otomatikleştirilmiş veya insan etkileşimi sağlayan makinelerin çalıştırılmasında kullanılabilmektedir [10]. Raspberry Pi mini bilgisayarı genellikle mikrokontrolcü kartların gücünün yetersiz kaldığı ve birden fazla işlemin eş zamanlı olarak yapılması gerektiği durumlarda tercih edilmektedir.

Raspberry Pi 3, Raspberry Pi ailesinin ilk 4 çekirdekli 64 bit işlemcili bilgisayarıdır. Raspberry Pi 3 içerisinde 1 GB RAM ve 400 $\mathrm{MHz}$ VideoCore IV grafik işlemci barındırmaktadır. İşlemcisi ise 64 bit veri yoluna sahip 1.2 GHz hızında ARM Cortex A53 çipidir. Sahip olduğu tüm özellikler Tablo 1'de listelenmektedir [11]. Şekil 1'de ise Raspberry Pi 3'ün yapısının genel bir görünümü yer almaktadır. 
Tablo 1. Raspberry Pi 3 'ün özellikleri

\begin{tabular}{l}
\hline Özellikler \\
\hline $1.2 \mathrm{GHz} 4$ çekirdekli 64-bit ARM Cortex-A53 işlemci \\
1GB RAM \\
400 Mhz 2 çekirdekli Videocore IV® Multimedia \\
Dahili WiFi - BCM43143 \\
Bluetooth 4.1 (Bluetooth Low Energy - BLE) \\
40 Adet GPIO \\
4 Adet USB 2 \\
4 uçlu Stereo çıkışı ve karmaşık video çıkışı \\
Full HDMI \\
RPi Kamera bağlantısı için CSI kamera portu \\
RPi 7" dokunmatik ekran için DSI ekran portu \\
Micro SD soketi \\
2,5A'e kadar destekli güç kartı \\
Güç ve aksiyon LED’i
\end{tabular}

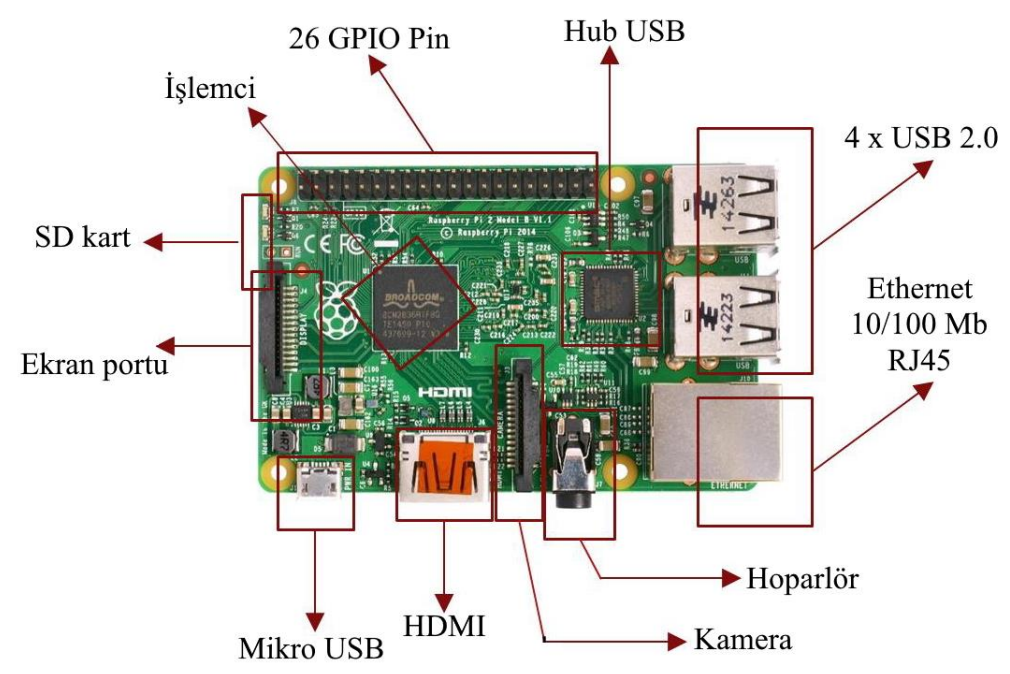

Şekil 1. Raspberry Pi 3'ün genel yapısı

Şekil 2'de Raspberry Pi 3 mini bilgisayarındaki genel amaçlı giriş-çıkış (GPIO - General-Purpose Input/Output), toprak (GND Ground), doğrudan sayısal kontrol (DNC - Direct Numerical Control) ve güç (3.3V ve 5V) pinleri gösterilmektedir [12].

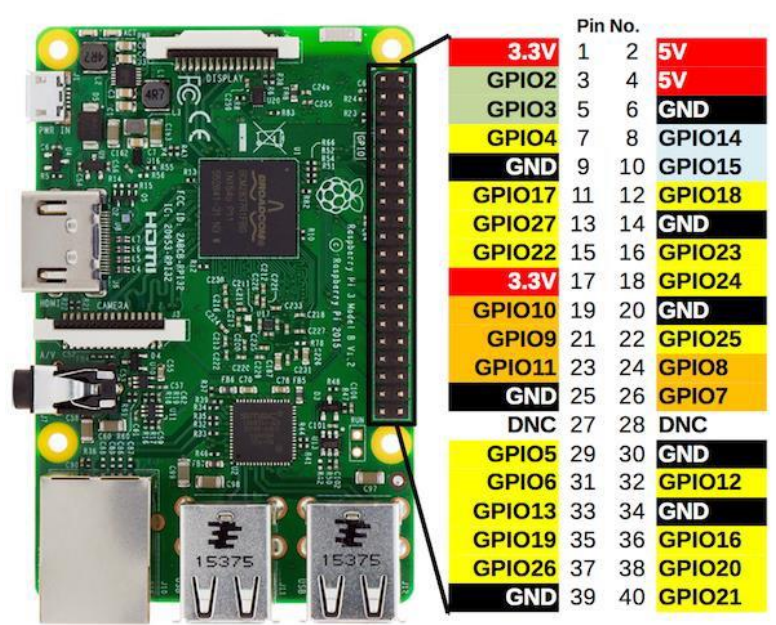

Şekil 2. Raspberry Pi 3 giriş çıklş pinleri 


\subsection{V1.3 Raspberry Kamera Modülü}

Çalışmada kullanılan Raspberry Kamera Modülü 1.3 Versiyonunda, kameranın üzerinde bulunduğu kartın boyutları $25 \times 20$ mm olup, kamera dâhil derinliği 9 mm'dir. 5 MP çözünürlüklü kamera üzerinde sabit odaklı bir lens bulunmaktadır. $2592 \times 1944$ piksel statik resim çözünürlüğü sunan kamera, video çekimlerinde ise 1080p30, 720p60 ve 640x480p60/90 çözünürlüğü desteklemektedir. Kamera modülü Raspberry Pi üzerinde çalışan tüm işletim sistemleri tarafından desteklenmektedir. Şekil 3 'te kamera modülünün bir görünümü yer almaktadır.

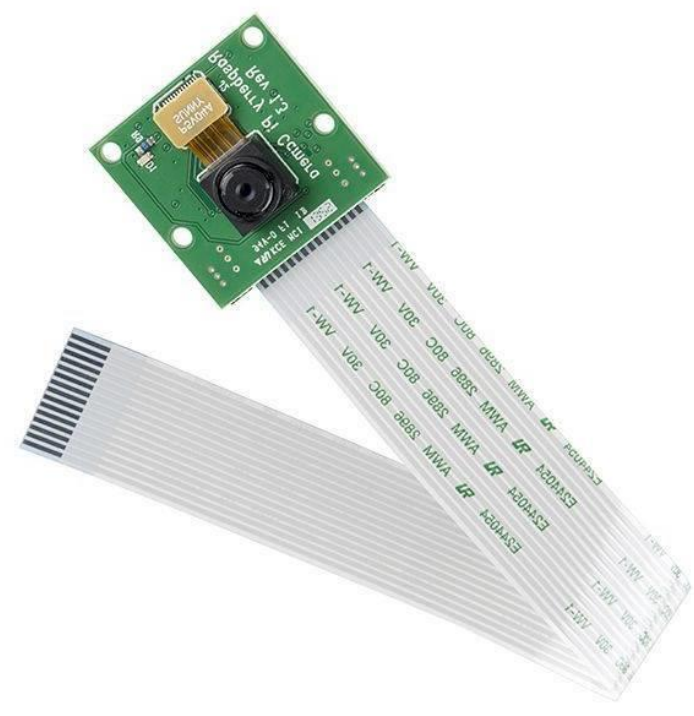

Şekil 3. Raspberry Kamera Modülü V1.3

\subsection{Kullanılan Basit Modüller}

Geliştirilen donanım içerisinde kutuya çöp atıldığını sisteme bildirmesi için 3 çift IR alıcı-verici kızılötesi LED (her kutu için 1'er adet) ve çöpün görüntüsünün kamera tarafından düzgün alınması için kutu içerisinin aydınlatılmasında kullanılmak üzere 3 adet LED kullanılmıştır. Ayrıca, bileşenlerin birleştirilebilmesi için devre kartı, LED’lerin fazla akımdan etkilenmemesi için 110 ohm'luk dirençler ve modüllerin bağlanması için jumper kablolar kullanılmıştır. İşlem sonuçlarının gösterilmesi için kutuların dışına bağlanacak 1 adet 16x2 LCD ekran ve LCD ekrandaki kablo fazlalığını ortadan kaldırmak için I2C modülü kullanılmıştır. Bir de Raspbian Stretch işletim sisteminin kurulabilmesi için MicroSD Kart (16GB) kullanılmıştır. Kullanılan basit modüllerin görüntüleri Şekil 4'te toplu olarak gösterilmektedir.

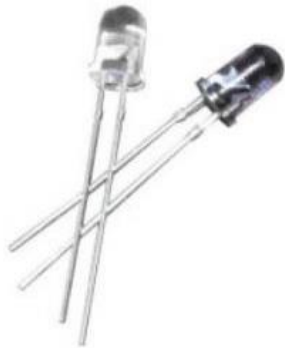

IR Alıcı-Verici Çifti (3 adet)

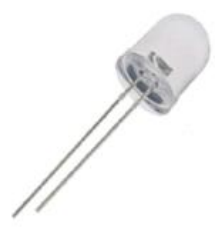

LED (3 adet)

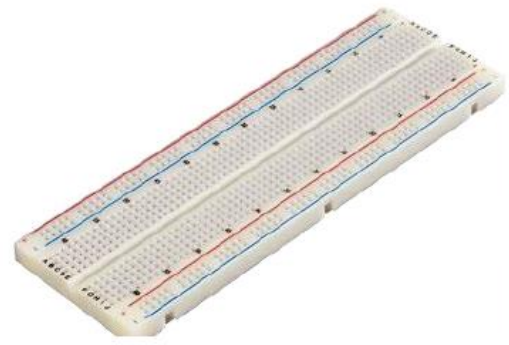

Devre Tahtas1

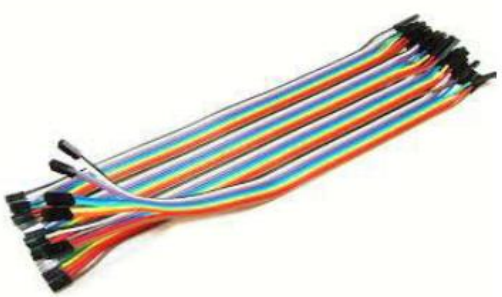

Jumper Kablolar

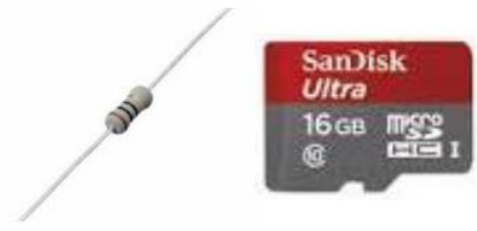

110 ohm'luk dirençler MicroSD Kart

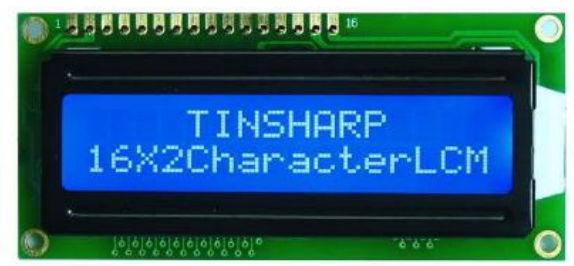

16x2 LCD Ekran

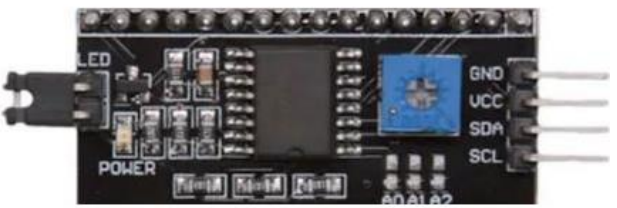

I2C Modülü

Şekil 4. Kullanılan basit modüller 


\subsection{Rasbian Stretch İşletim Sisteminin Kurulması}

Raspberry Pi’ye kendi işletim sisteminin kurulması için bir adet 16 GB MicroSD Kart kullanılmıştır. İşletim sisteminin kurulumunu yapmak için öncelikle SdCardFormatter uygulaması [13] ile MicroSD Kart biçimlendirilmektedir. Raspbian Stretch işletim sistemi indirilerek MicroSD Karta yüklenmesi için Win32 uygulaması [14] kullanılmıştır. Raspbian Stretch işletim sistemi [15], Win32 uygulaması kullanılarak MicroSD Karta yüklenmiş ve işlem tamamlanmıştır.

Windows işletim sistemi SSH bağlantısını desteklemediğinden, Raspberry işletim sistemine uzaktan bağlantı sağlamak ve oturum açabilmek için Putty [16] yazılımı bilgisayara indirilmiştir. Linux işletim sistemlerinde bulunan grafik arabirimini Windows işletim sistemi üzerinde çalıştırmak için ise Xming [17] programı bilgisayara kurulmuştur.

\subsection{Raspberry Pi 3'e Uzaktan Bağlanma}

Monitör (tv, masaüstü bilgisayar monitörü vs.) kullanılarak bağlantısı gerçekleştirilen Raspberry'de, Raspberry ayarlarından SSH’ı 'enabled' ettikten sonra, MicroSD Kart içindeki 'cmdline.txt' dosyası içerisine bilgisayarın IP adresinin ilk üç kısmı aynı olacak şekilde bir IP adresi tanımlanır. Böylelikle Raspberry Pi’ye bir IP adresi atanmış olmaktadır.

IP adresi atandıktan sonra MicroSD Kart Raspberry Pi'ye takılıp, Ethernet kablosu kullanılarak bilgisayarla bağlantı kurulmuştur [18]. 'Putty' uygulamasında, Bilgisayar adı (Host Name) (ya da IP adresi) kısmına Raspberry Pi için atanan IP adresi girilip, Connection Type'1 'SSH' olarak seçilir. X11 forwarding aktif edildikten sonra 'open' komutu ile Raspberry Pi ve bilgisayar arasında bağlantı sağlanabilmektedir. 'Xming' ara yüz uygulaması açılarak, Putty’nin konsol ekranında Raspberry Pi’ye varsayılan (default) olarak gelen kullanıcı adı ve şifresi ile girildikten sonra 'startlxde' komutuyla Raspberry Pi masaüstü ekranına erişim sağlanmaktadır.

Kablosuz bağlantı yapmak için ise; Raspberry Pi'nin Wi-Fi özelliğinden yararlanılarak Raspberry Pi’nin bağlı olduğu internetin IP'sinden, dolayısıyla bilgisayarın bağlı olduğu ağın IP adresinden yararlanılarak Putty uygulamasında IP adresi yerine yazılıp bağlantı yapılabilir. Böylelikle Ethernet kablosu gerekmeden Raspberry Pi’nin masaüstüne uzaktan bağlantı yapılabilmektedir.

\subsection{Donanım için Gerekli Bağlantıların Yapılması}

Kızılötesi alıcı-verici LED’lerin devresi Şekil 5’teki gibi kurularak alıcı-verici LED'lerinin iletişimde olup olmadığının anlaşılması amacıyla LED devresi kurularak gerekli kod yazılmıştır. Alıcı-verici LED’ler iletişim halindeyken LED’in sönmesi ve iletişimin kesilmesiyle (yani çöp atıldığında) beyaz ışık yayan LED'in yanması sağlanmıştır.

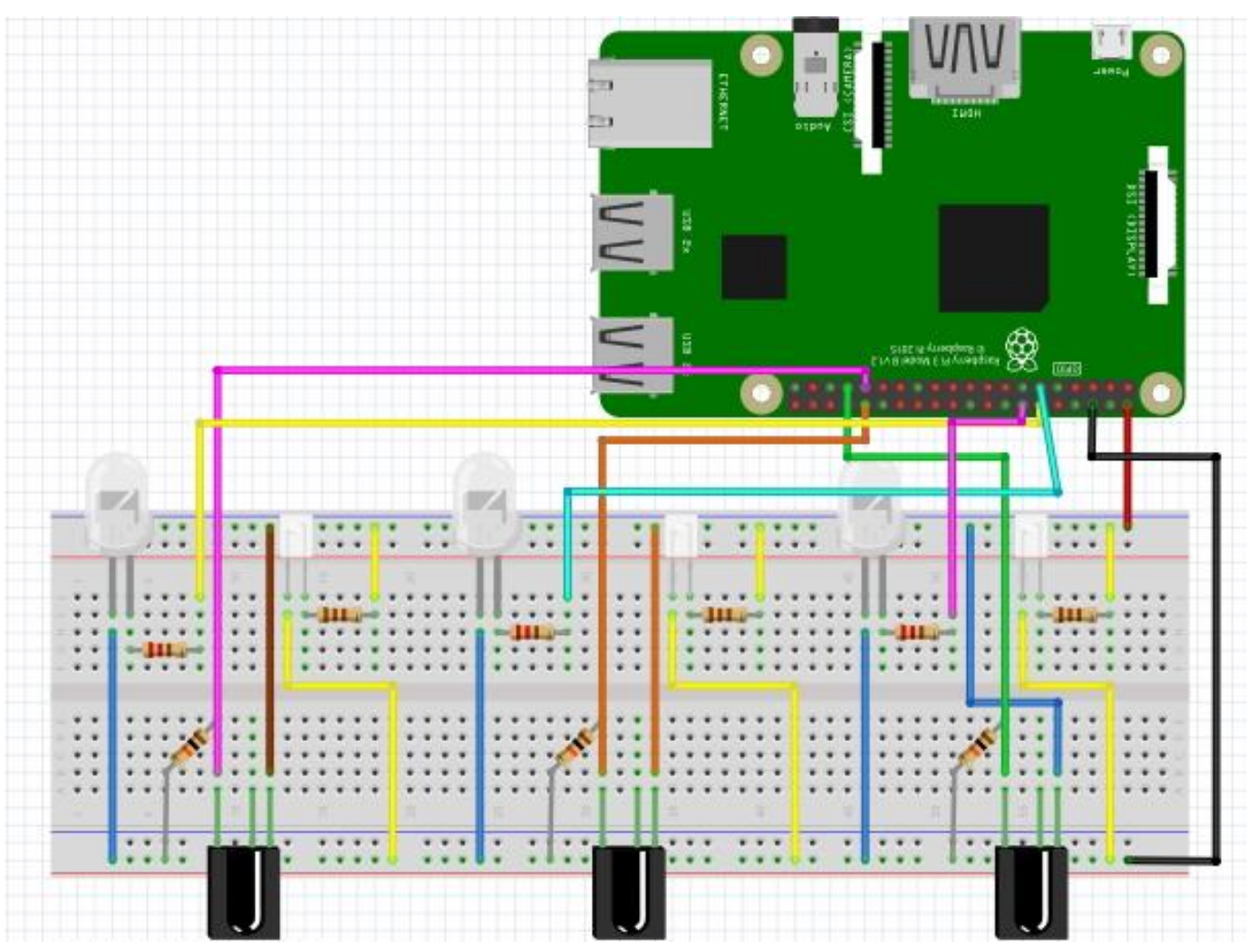

Şekil 5. Kızılötesi alıcı-verici LED bağlantıları

Şekil 6 (a)'da donanımın kutular üzerindeki yerleşimi, Şekil 6 (b)'de donanımın jumper kablolar ile bağlanma şekilleri ve Şekil 6 (c)'de ise kutu içerisine çöp atılma esnasında IR alıcı vericilerin çöpü algılayıp LED'i aktif hale getirerek yakması görülmektedir. 


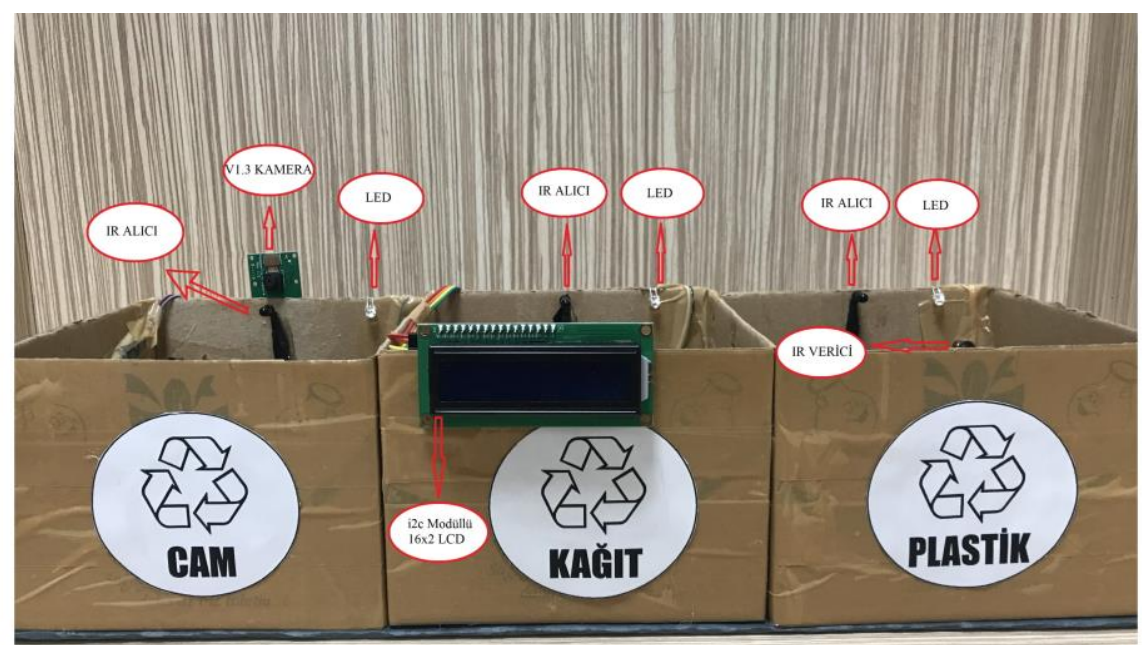

(a)

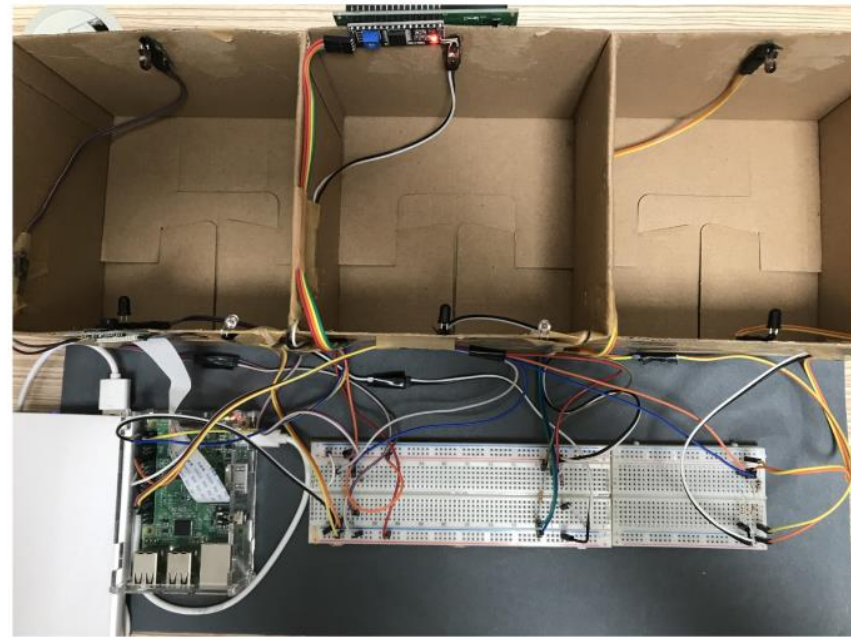

(b)

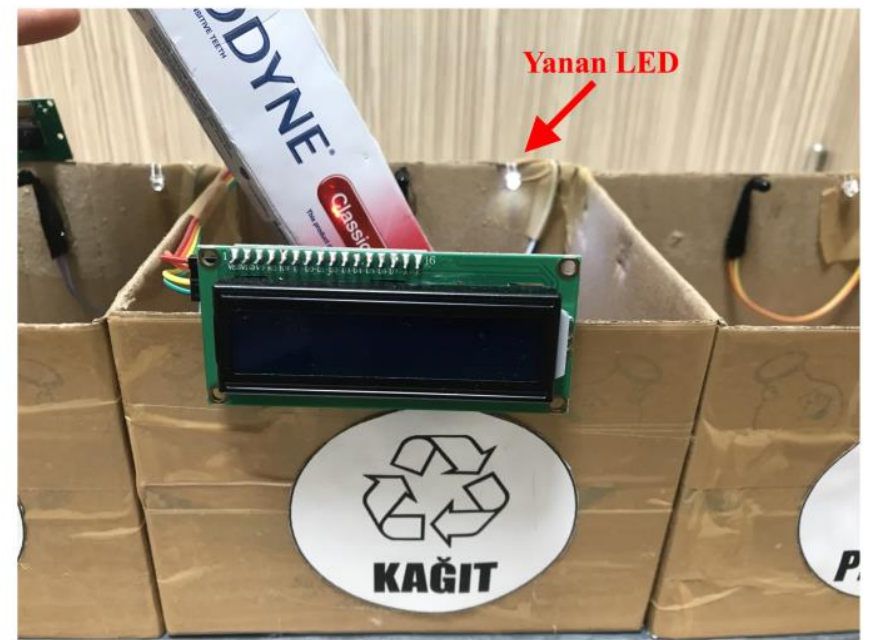

(c)

Şekil 6. Donanımın kutular üzerindeki yerleşimi (a), donanımın jumper kablolar ile bağlanma şekilleri (b) ve kutu içerisine çöp atılma esnasında IR alıcı vericilerin bunu algılayıp LED'i aktif hale getirerek yakması (c)

\subsection{Gerekli Yazılımların Kurulumu}

Geliştirilen donanımın amacına uygun olarak, nesneleri tanıması için gerekli olan görüntü işleme araçları bu çalışmada OpenCV kütüphanesi ile kullanılmıştır [19]. Windows işletim sisteminde görüntü işleme ile ilgili testlerin önceden yapılabilmesi için Python 3.5.6 [20]; Anaconda 3.5.2 [21] ve Pycharm [22] programları kurulmuştur.

Kurulumlardan sonra OpenCv Kütüphanesi; Python için komut isteminde, Anaconda için Navigator IDE’si içinde, Pycharm için kendi IDE'si içinde indirilerek kullanıma hazır hale getirilmiştir. Pycharm üzerinde programlar yazılırken Anaconda ile bağlantı kurulmuş ve programların Anaconda ile yapılandırılması ve çalıştırılması sağlanmıştır. Bunlarla birlikte Raspberry Pi 3 üzerine de OpenCV Kütüphanesi yüklenmiştir [23].

Çalışmanın bu aşamasından sonra, Google’ın açık kaynak kodlu derin öğrenme (deep learning) kütüphanesi olan Tensorflow üzerinde çalışılmıştır. Tensorflow [24], Raspberry’e başarılı bir şekilde yüklenerek kullanıma hazır hale getirilmiştir.

\subsection{Eğitim ve Test için Verilerin Hazırlanması}

Çalışmada; cam, plastik ve kâğıt maddelerinden yapılmış malzemeleri ayırt etmek için bir derin öğrenme sınıflandırıcısı oluşturulmuştur. Bunun için kullanılan malzemelerin yer aldığı 845 adet fotoğraf çekilmiş ve fotoğraflar aynı boyuta (500x400 piksel) getirilmiştir. Sınıflandırıcının oluşturulması için öncelikle; her bir fotoğrafa içerdiği nesnenin maddesini ve nesnenin görüntü içerisindeki koordinatlarını belirten etiketler ('.xml' dosyaları) hazırlanmıştır. Böylelikle her fotoğrafın eğitilmesi için '.xml' dosyaları oluşturulmuştur. Hazırlanan görüntülerden, örnek olarak bazıları Şekil 7'de gösterilmektedir. 

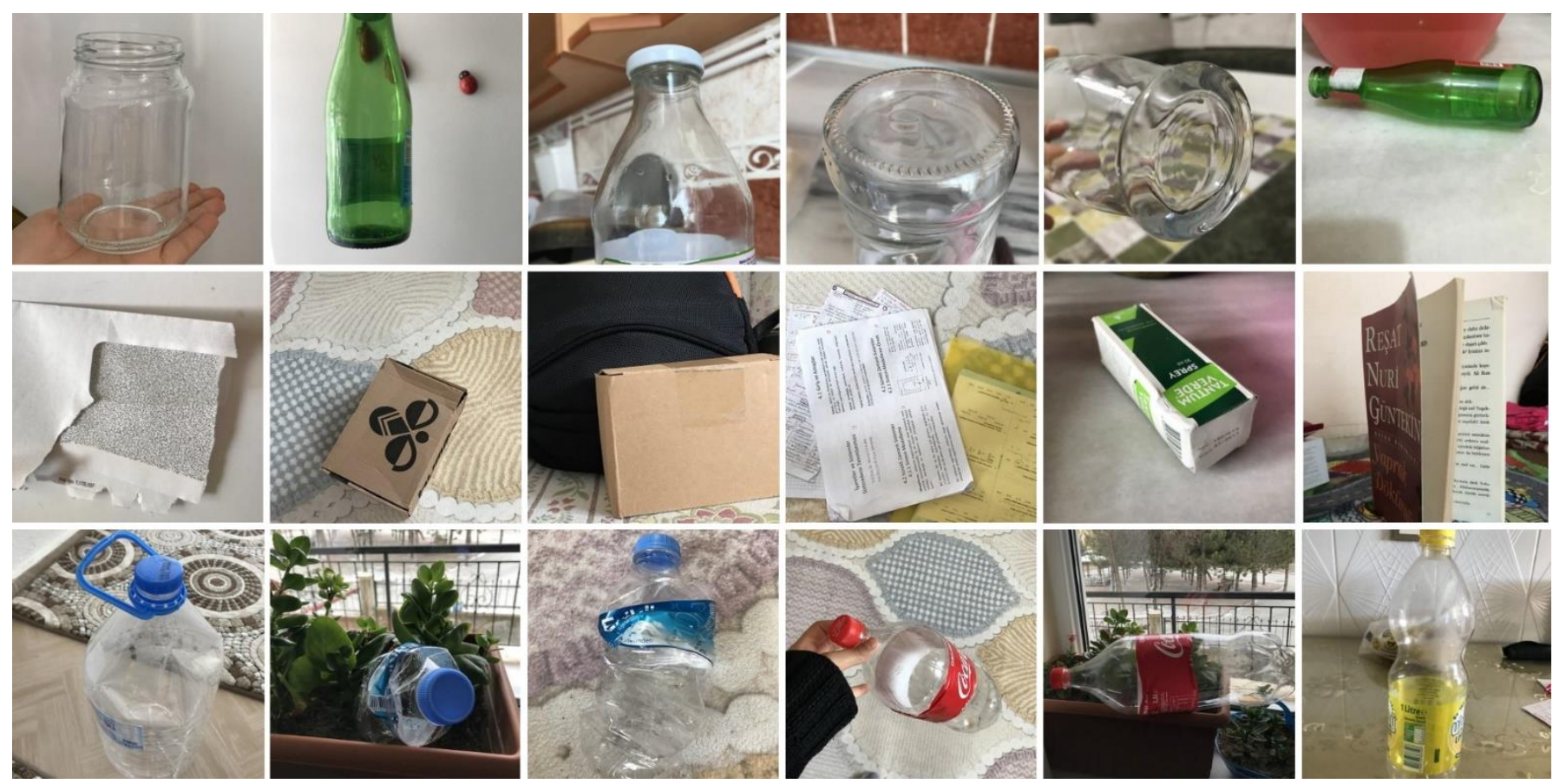

Şekil 7. Eğitim verisi için hazırlanan resimlerden bazı örnekler

\section{Test Sonuçları}

Eğitim sürecinin CPU'da daha yavaş olması nedeniyle, yüksek özelliklerde bir GPU'ya ihtiyaç duyulmuş; bu yüzden eğitimi tamamlamak üzere, ücretsiz GPU desteği sunan Google Colab kullanılmıştır [25]. Google Colab üzerinde eğitimi yapabilmek [26] için sırasıyla; 'Google Drive'da bir klasör yaratılarak, klasörün içinde 'Google Colaboratory' dosyası oluşturulmuştur. Google Drive ile gerekli bağlantılar sağlandıktan sonra 'Tensorflow/models' github klasörü indirilerek gerekli derlemeler yapılmıştır. “.LabelImg” ile etiketlenerek oluşturulan '.xml' dosyaları da kullanılarak gerekli eğitim kodları yazılmıştır.

Hazırlanan 845 adet resimden 662 tanesi eğitim için kullanılmış, kalan 183 tanesi de test amaçlı kullanılmıştır. Eğitim, Google Colab'ın GPU'yu kullanılmaya izin verdiği 12 saat içerisinde gerçekleştirilmiştir. Eğitim, yaklaşık olarak 10 saatte ve 73500 adımda sonlandırılmıştır. Eğitim sonucunda gerekli eğitim modeli kullanıma hazır hale getirilmiştir. Windows'ta, Tensorflow ile nesne tanımlama işlemi için Anaconda Prompt’ta sanal ortam kurularak Tensorflow kurulumu [24] gerçekleştirilmiştir. Google Colab üzerinde eğitilen model indirilmiş ve test amaçlı olarak Windows üzerinde bilgisayar kamerası ile nesne tanıma işlemi gerçekleş̧irilmiştir. Hazırlanan 662 eğitim verisi ile eğitilmiş derin öğrenme modelinin, hazırlanan 183 test verisi üzerindeki başarı sonucu \% 75,4 olmuştur. Şekil 8'de, eğitim sonunda elde edilen modelin bazı örnekler üzerindeki başarı sonuçları görülmektedir.
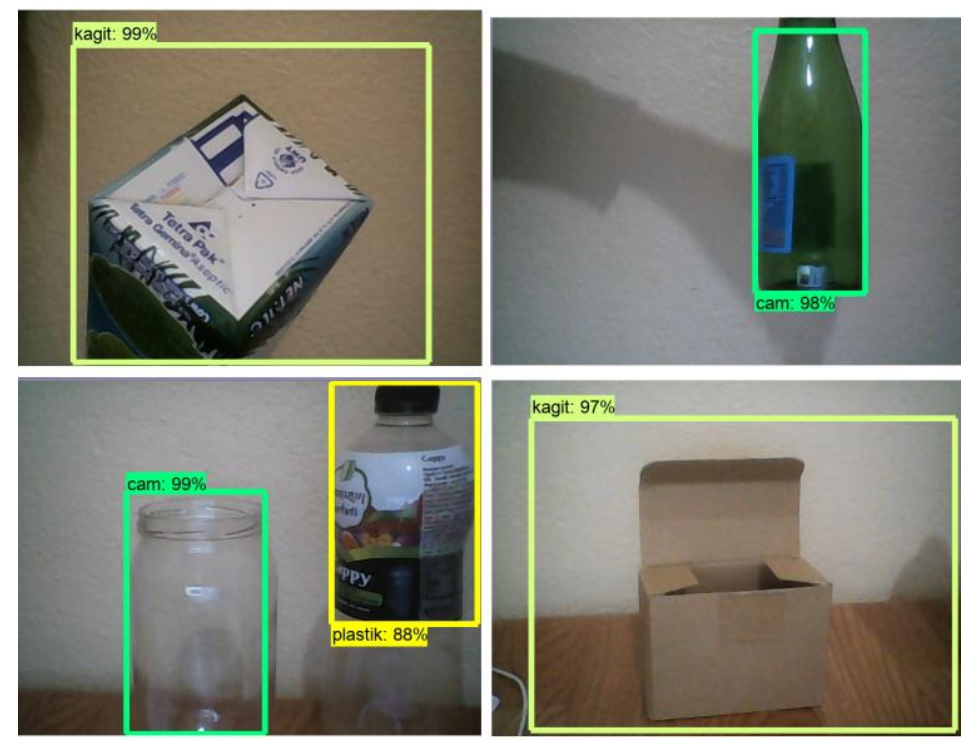

Şekil 8. Eğitim sonunda elde edilen modelin bazı resimler için verdiği sonuç olasıllı değerleri 
Raspberry Pi'de nesne tespitinin denenmesi için, eğitilmiş model Raspberry Pi’ye yüklenip; koddaki gerekli ayarlar değiştirilerek derlenmiştir. Yazılım yüklemesi de hazır hale gelen sistem en son olarak kutu içerisine malzemeler atılarak test edilmiş ve Şekil 9'da görüldüğü gibi LCD ekran çıktıları elde edilmiştir. LCD ekranda tanımlanan nesnenin hangi sınıfa ait olduğu ve kullanıcıya aktarılması için daha önceden atığın cinsine göre belirlenmiş olan bakiye miktarı gösterilmektedir.
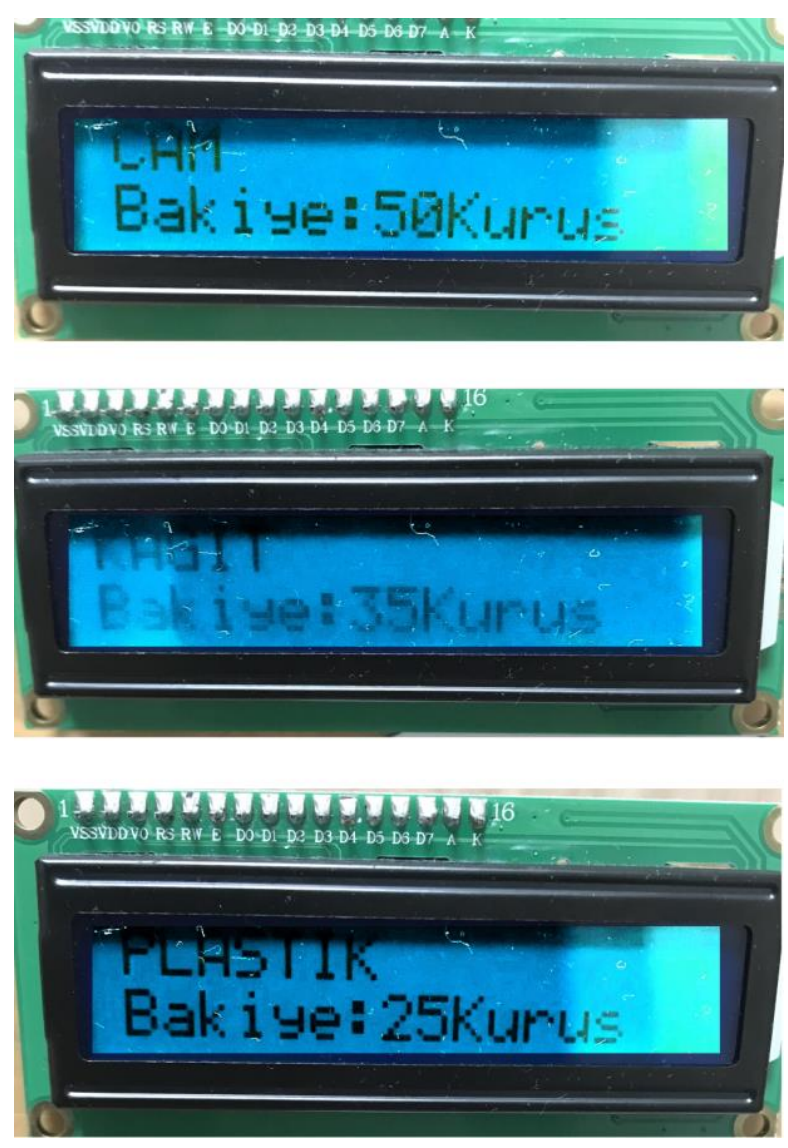

Şekil 9. Geliştirilen donanım üzerinde yapılan bazı testler sonucundaki LCD ekran çıktıları

\section{Tartışma}

Her dakika, en az 15 ton plastik atık okyanusa sızmaktadır ve bu bir çöp kamyonunun kapasitesine eşdeğerdir. Plastik şişeler ve kutular gibi atıklar yüzünden ölüm tehlikesi yaşayan deniz canlıları ile kâğıt ihtiyacı için tüketilen milyonlarca ağaç, tüm canlılar için tehlike oluşturmaktadır. Bu sonsuz tüketim faaliyetlerinde yapılacak en anlamlı çalışma, tüketim israfını en aza indirgemek ve atıkların geri dönüşümünü sağlamaktır. Geliştirilen donanımın profesyonel şekilde hayata geçirilmesi ile nesnelerin tanınması ve böylece atık ayrımının yapılabilmesi sağlanabilecektir. Projede bakiye transferinin sağlanmasıyla da tüketiciyi geri dönüşüme teşvik etmek kolaylaşacaktır. Bu sayede hem üretici hem tüketici olumlu yönde etkilenecek ve doğal hayatın zarar görmesinin önüne geçilmesi sağlanacaktır.

Gelecek çalışmalar olarak, geliştirilen donanımın gerçek hayatta kullanımını sağlayacak gelişmiş hali olarak deney tahtası ve jumper kablolar yerine entegre devreler kullanılabilir ve kullanıcının hesabı ile etkileşime geçebilecek manyetik kart okuyucu modülleri sisteme dahil edilebilir. Ayrıca, hazırlanan derin öğrenme eğitim modeli için kullanılan eğitim verileri, donanımın gerçek hayatta kullanım şeklindeki görüntülerden hazırlanarak daha akıllı hale getirilebilir. Eğitim için kullanılan görüntü sayısı arttırılarak, derin öğrenme modelinin daha iyi eğitilmesi sağlanarak, test aşamasında daha iyi sonuçlar elde edilebilir. Bunun dışında ekran çıktısının yanında hoparlörler kullanılarak ses yardımı ile de kullanıcıya işlem sonucu ile ilgili bilgi verebilir.

\section{Sonuç}

Geliştirilen donanım geri dönüşebilir atıklardan cam, plastik ve kâğıt atıkları yapay zekâ desteği ile belirli bir başarı oranında tanıyabilmektedir. Proje, örnek olarak belirlenen atıkların ayrımını yapabilmekte ve çıktı olarak da belirlenen bakiye transferini sanal olarak yapabilmektedir. Donanım basit malzemeler kullanılarak yapılmıştır ve elektronik malzemelerin gerekli bağlantılarının yapılması ile görüntü işleme yazılımı ile etkileşim halinde ekran sonuçları vermektedir. Geliştirilen prototip, donanımsal olarak doğru bir şekilde çalışmayı başarmıştır. Hazırlanan eğitim verileri ile oluşturulan yazılım kısmında ise test verilerinde \% 75,4 başarı sağlanmıştır. 


\section{Kaynakça}

[1] Gündüzalp, A.A., Güven, S., (2016). Atık , Çeşitleri , Atık Yönetimi , Geri Dönüşüm ve Tüketici : Çankaya B elediyesi ve Semt Tüketicileri Örneği. Hacettepe Üniversitesii Sosyolojik Araştırmalar E-Dergisi.

[2] European-Plastics, (2017). An analysis of European plastics production, demand and waste data. Plastics - the Facts. doi: 10.1016/j.marpolbul.2013.01.015.

[3] Yüce, E., Kılıç, M., (2014). PVC ve PET Atıkların Seçimli Flotasyonu Bölüm 1: Plastikler, Çevresel Etkileri, Geri Dönüşümü. Çukurova Üniversitesi Mühendislik-Mimarlık Fakültesi Dergisi. doi: 10.21605/cukurovaummfd.242821.

[4] Güleç Solak, S., Pekküçükşen, Ş., (2018). Türkiye'de kentsel katı atık yönetimi: karşılaştırmalı bir analiz. Karamanoğlu Mehmetbey Üniversitesi İktisadi Ve İdari Bilimler Fakültesi.

[5] Yücel, K., Türkiye’de Katı Atık Yönetimi ve Geri Kazanımı, Yıldız Teknik Üniversitesi, 1997.

[6] Geleri, E., Geri dönüşümün çevreye etkilerine ilişkin öğrenci tutumları, Fırat Üniversitesi, 2019.

[7] Özbay, Ş., Fen ve teknoloji programı içinde kompost hakkında verilen ettkinliklerin öğrencilerin akademik başarılarına ve çevreye tutumlarına etkisi, Çanakkale Onsekiz Mart Üniversitesi, 2010.

[8] Kaçtıŏglu, S., Şengül, Ü., (2010). Erzurum kenti ambalaj atıklarının geri dönüşümü için tersine lojistik ağı tasarımı ve bir karma tamsayılı programlama modeli. Atatürk Üniversitesi İktisadi ve İdari Bilimler Dergisi. 24(1). ss. 89-112. doi: 10.16951/iibd.09418.

[9] Alakaş, H.M., Kızıltaş, Ş., Eren, T., Özcan, E., (2018). Sıfır atık projesi kapsamında atıkların toplanması: Kırıkkale ilinde homojen çok araçlı araç rotalama uygulaması. Harran Üniversitesi Mühendislik Dergisi. 3(3). ss. 190-196.

[10] Avcu, M., Raspberry Pi 3 ile güneş takip sistemi (RPiGTS), İstanbul Arel Üniversitesi, 2018.

[11] Direnc.net, Raspberry Pi 3 Model B, 2020. [Çevrimiçi]. Available at: https://www.direnc.net/raspberry-pi-3.

[12] Raspberry Pi GPIO Programming in C, BMOW, 2018. [Çevrimiçi]. Available at: https://www.bigmessowires.com/2018/05/26/raspberry-pi-gpio-programming-in-c/.

[13] Association, S., SD Memory Card Formatter. [Çevrimiçi]. Available at: https://www.sdcard.org/downloads/formatter/.

[14] SourceForge, Win32 Disk Imager. [Çevrimiçi]. Available at: https://sourceforge.net/projects/win32diskimager/.

[15] Debian, 2017-11-29-Raspbian-Stretch. [Çevrimiçi]. Available http://debian.rutgers.edu/raspbian_images/raspberrypi/images/raspbian/2017-11-29-raspbian-stretch/.

[16] Tatham, S., PuTTY, 2020. [Çevrimiçi]. Available at: https://www.chiark.greenend.org.uk/ sgtatham/putty/latest.html.

[17] Harrison, C., Xming X Server for Windows, 2016. [Çevrimiçi]. Available at: https://sourceforge.net/projects/xming/.

[18] Raspberry Pi Türkiye Topluluğu, Raspberry Pi'ye ağ kablosu ile doğrudan bağlanma. [Çevrimiçi]. Available at: https://www.raspitr.com/2014/10/12/raspberry-piye-ag-kablosu-ile-dogrudan-baglanma/.

[19] Pişkin, M., OpenCv Video Eğitim Serisi, 2016. [Çevrimiçi]. Available at: http://mesutpiskin.com/blog/opencv-egitim-serisi.html.

[20] Python, Python 2.7.13, 2016. [Çevrimiçi]. Available at: https://www.python.org/downloads/release/python-2713/.

[21] Archive, A., Anaconda Installer Archive, 2020. [Çevrimiçi]. Available at: https://repo.anaconda.com/archive/.

[22] JetBrains, Download Pycharm, 2020. [Çevrimiçi]. Available at: https://www.jetbrains.com/pycharm/download/.

[23] Yazılım Akademi, Raspberry Pi \& Python OpenCv Kurulum, 2017. [Çevrimiçi]. Available at: https://www.youtube.com/watch?v=j5echA62YM8.

[24] EdjeElectronics, Tensorflow-Object-Detection-on-the-Raspberry-Pi, 2019. [Çevrimiçi]. Available at: https://github.com/EdjeElectronics/TensorFlow-Object-Detection-on-the-Raspberry-Pi.

[25] Medium, Google Colab Free GPU Tutorial, 2018. [Çevrimiçi]. Available at: https://medium.com/deep-learning-turkey/googlecolab-free-gpu-tutorial-e113627b9f5d.

[26] Medium, Training an Object Detection Model with Tensorflow API using Google COLAB, 2019. [Çevrimiçi]. Available at: https://medium.com/analytics-vidhya/training-an-object-detection-model-with-tensorflow-api-using-google-colab$4 \mathrm{f} 9 \mathrm{a} 688 \mathrm{~d} 5 \mathrm{e} 8 \mathrm{~b}$. 